\title{
Linx
}

Revue des linguistes de l'université Paris X Nanterre

$77 \mid 2018$

Regards croisés sur le futur en français et dans différentes langues romanes

\section{Remarques sur le futur en sardo logudorese}

\section{Franck Floricic}

\section{(2) OpenEdition \\ Journals}

Édition électronique

URL : http://journals.openedition.org/linx/2871

DOI : 10.4000/linx.2871

ISSN : 2118-9692

\section{Éditeur}

Presses universitaires de Paris Nanterre

\section{Édition imprimée}

Date de publication : 30 octobre 2018

Pagination : 185-208

ISBN : 978-2-84016-329-9

ISSN : 0246-8743

Référence électronique

Franck Floricic, «Remarques sur le futur en sardo logudorese », Linx [En ligne], 77 | 2018, mis en ligne le 30 avril 2019, consulté le 03 mai 2019. URL : http://journals.openedition.org/linx/2871 ; DOI :

10.4000/linx.2871

Ce document a été généré automatiquement le 3 mai 2019.

Département de Sciences du langage, Université Paris Ouest 


\title{
Remarques sur le futur en sardo logudorese
}

\author{
Franck Floricic
}

« (...) nec proprie dicitur: tempora sunt tria, praeteritum, praesens et futurum, sed fortasse proprie diceretur: tempora sunt tria: praesens de praeteritis, praesens de praesentibus, praesens de futuris. Sunt enim haee in anima tria quaedam et alibi ea non video, praesens de praeteritis memoria, praesens de praesentibus contuitus, praesens de futuris expectatio » (Saint Augustin, Les confessions. Livre XI)

(« On ne peut pas dire, à proprement parler, qu'il y a trois temps : le passé, le présent et le futur ; mais peut-être dirait-on avec plus de justesse : il y a le présent des choses passées, le présent des choses présentes, le présent des choses futures. Ces trois temps existent en effet dans l'âme, et je n'en vois pas ailleurs : le présent des choses passées, c'est leur souvenir; le présent des choses présentes c'est leur intuition; le présent des choses futures, c'est leur attente »)

\section{Introduction}

L'objectif de cette contribution est d'offrir une description du futur en sardo logudorese. $\mathrm{Si}$ le futur a donné lieu à de nombreuses études du point de vue typologique, il n'en va pas de même en sarde où, à l'exception notamment de Pisano (2007), (2009) et de Loporcaro (1999), on ne peut pas dire que la problématique en question ait attiré l'attention de nombreux chercheurs. On se proposera donc dans les lignes qui suivent de présenter en premier lieu quelques-unes des caractéristiques fonctionnelles et sémantiques 
essentielles du futur et de sa genèse. On présentera ensuite, sur la base de données dialectologiques existantes (les atlas linguistiques) et d'enquêtes de terrain réalisées en Sardaigne, quelques-unes des spécificités morpho-phonologiques de la formation du futur dans la variété logudorese parlée à Buddusò (Provincia Olbia-Tempio). On verra que pour exprimer le futur cette variété recourt à une construction pan-sarde de nature périphrastique du type "J'ai à + verbe à l'infinitif ». On verra également que d'autres constructions périphrastiques sont attestées en sarde, qui posent naturellement la question de l'analycité telle qu'elle se manifeste dans cette variété.

\section{Aspects sémantiques et fonctionnels}

2 A l'origine, le futur est un prospectif ou un désidératif (cf. Meillet 1948: 145): le locuteur envisage, désire, souhaite l'accomplissement ou la réalisation d'un état de fait postérieur au moment de l'énonciation : le futur est donc fondamentalement lié à la modalité et à la volition.

3 Du point de vue formel le futur roman provient de la synthèse du verbe lexical et du verbe habère, comme l'avait vu pour la première fois Antônio de Nebrija dans sa Gramática Castellana de 1492 : «El venidero del indicativo dizese por rodeo del presente del infinitivo $i$ del presente del indicativo deste verbo $e$ as, i assi dezimos io amare como si deixessemos io e de amar " (GC, 125)1.

4 La formation du futur roman est donc un exemple de grammaticalisation ou de réanalyse (cf. Meillet 1948: 145; Klausenburger $2000: 77$, etc.): le verbe habēre perd sa valeur lexicale (i.e. le sens de possession) pour acquérir une valeur strictement grammaticale ${ }^{2}$; dans ce type de futur, le sujet de l'auxiliaire contrôle le sujet de la complétive infinitivale. Il s'agit d'une construction désidérative ou déontique dans laquelle le sujet envisage comme souhaité ou nécessaire l'état de fait décrit par la complétive infinitivale.

5 Comme l'écrit Kuryłowicz (1975a: 13) dans ses Esquisses linguistiques, « The future is due to an analogous semantic development: I shall write, you will write etc. had originally the value "I ought to write ", " you want (desire) to write » and so on. But if the obligation or the desire of a certain action is synchronous with the act of speaking, then the action itself can take place only afterwards, hence the development « present obligation, desire of action » to « future action ».

6 L'enracinement du futur dans le hic et nunc du moment de locution $t_{0}$ explique donc aussi les liens entre présent et futur et la possibilité d'utiliser le présent en lieu et place d'un futur dédié (cf. demain je prends le train pour Paris) - Benveniste (1966:245) observait d'ailleurs que le futur «n'est qu'un présent projeté vers l'avenir ». On notera à cet égard qu'une langue comme le kurde sorani ne possède pas de futur et utilise à cette fin le présent, contrairement au kurde kurmandji (cf. Thackston $2006: 26$, note 1). Le kurde badini recourt quant à lui à la particule dê. L'arménien classique ne possède pas non plus de futur et recourt à cet effet au subjonctif, et l'on pourrait multiplier les exemples qui illustrent l'instabilité et le renouvellement permanent des formes de futur.

\section{Aspects morphologiques}

7 La construction romane constitue donc un tour expressif qui avait toutes les chances de se substituer au futur synthétique latin amābo «j'aimerai» ( * *am-a-bhwo < *bhū « je suis 
/ deviens » (cf. ich werde lieben «j'aimerai »), amābis «tu aimeras», etc. Ce dernier en effet ne survit pas dans les langues romanes (cf. Lausberg 1971, II : p.214, § 837), si ce n'est que d'une manière résiduelle. D'autant qu'en outre le futur était morphologiquement peu transparent: à la première personne du singulier le subjonctif et le futur sont syncrétiques (cf. Bonfante 1998: 107; Klausenburger 2000: 40, etc.). D'autre part, l'exposant du futur est le morphème - $b$ - aux première et deuxième conjugaisons latines, dont la voyelle thématique est longue (cf. amābit «il / elle aimera», tenēbit «il / elle tiendra ») mais -e- aux troisième et quatrième conjugaisons : perdĕt « il / elle perdra ",

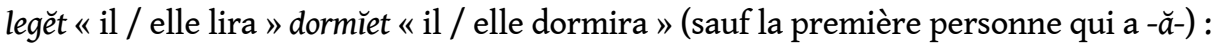

(1)

$$
\text { a.b.c. }
$$

Présent Futur Subjonctif

lego legăm legăm

leğs legēs legās

leğt legĕt legăt

legřmŭs legēmus legāmus

leğtris legētis legātis

legŭnt legěnt legănt

Si l'on garde à l'esprit que l'occlusive bilabiale était destinée à s'affaiblir $(b>v$, d'où la confusion du futur amābit et du parfait amāuit) et qu'en outre les $\breve{\imath}$ et $\breve{e}$ atones aboutissaient tous deux à [e] / [ $\varepsilon]$, des formes telles que la 3sg du présent de l'indicatif perd̆̌t « il / elle perd » et la 3sg du futur perdĕt étaient destinées à devenir homophones, et l'échange des deux finales est parfaitement bien documenté dans les textes (cf. Lausberg 1971 : §837, p.214 ; Herman 2006 : 78-79, etc.). Or il s'agissait là d'une zone d'instabilité d'autant plus préjudiciable que la troisième personne du singulier est particulièrement fréquente (cf. sur cet aspect les nombreux travaux de Witold Mańczak). Qu'il suffise de penser également à la proximité formelle du futur et de l'imparfait de l'auxiliaire être : erō , erǐs, erĭt, erĭmus, erĭtis, erŭnt. (futur) en face de erăm, erās, erăt, erāmus, erātis, erănt (imparfait).

9 Il pourrait sembler étrange que les langues romanes n'aient pas développé une construction qui était disponible en latin, en l'occurrence le futur périphrastique en -urus sum (cf. Lausberg 1971: \$843, p.216). D’après Herman (2006: 84sqq.) cette construction était particulièrement fréquente dans les textes tardifs et constituait donc un candidat idéal pour le renouvellement du futur. Comme le souligne Herman (op. cit.), cependant, le verbe habēre offrait l'avantage de commencer par une voyelle et de s'agglutiner peut-être plus facilement à l'infinitif. Naturellement, cet aspect des choses ne peut être qu'effleuré ici et mériterait une investigation plus poussée.

Diverses stratégies ont donc été développées dans les langues pour renouveler le futur et lui donner une nouvelle expression. Le roumain recourt au verbe a vrea «vouloir » et continue donc une structure du type vŏlěo cantare > vóiu cîntá (cf. Lausberg 1971: \$840, 215), de même d'ailleurs que le franc-comtois (cf. Herzog 1906: 67). On notera que la formation d'un néo-futur au moyen du verbe "vouloir » est considérée depuis au moins Miklosich (1861) comme une propriété aréale qui unit le roumain au bulgare, au serbocroate, au grec, au macédonien, à l'albanais, etc. (Tomić $2006: 1,5)$ :

(2)

a. voi cânta (Roumain)

will.1Sg.Mod.Cl sing.Inf

«I will sing"

b. Ja ću pevati / pevaću (Serbe) (NB. ću < hóću « je veux ») 
I will.1Sg.Mod.Cl sing.Inf/ sing+will.1Sg.Mod.Cl

«I will sing "

c. do të shkruaj (Albanais)

will.Mod.Cl Subj.Mark write.1Sg

«I will write "

d. va-s skriu (Roumain)

will.Mod.Cl-Subj.Mark write.1Sg

«I will write"

e. $\theta \mathbf{a}^{3} \gamma \rho \alpha \varphi \omega$ (Grec Moderne)

will.Mod.Cl write.1Sg

«I will write"

f. šte piša (Bulgare)

will.Mod.Cl write.1Sg

«I will write"

g. Ḱe pišam (Macédonien)

will.Mod.Cl write.1Sg

«I will write »

11 Les verbes de mouvement peuvent eux aussi être recyclés pour exprimer des valeurs aspectuo-temporelles particulières. $\mathrm{Du}$ reste c'est un verbe de mouvement que le sursilvain a exploité pour renouveler l'expression du futur qui y présente la forme venio ad cantare (cf. jeu végnel a cantar " je chanterai ») (cf. Lausberg, op. cit.) ${ }^{4}$. Ainsi en français aussi aller et venir de + infinitif ne sont-ils plus utilisés avec la valeur de verbe de mouvement, mais avec une valeur de futur ou de passé proches : cf. je vais prendre l'avion; je viens de prendre l'avion (cf. Lausberg 1971 : 212). La preuve que la valeur de motion s'est étiolée et que la construction analytique s'est grammaticalisée est que ces expressions périphrastiques admettent à leur tour l'insertion d'un verbe de mouvement : je vais aller prendre l'avion; je viens d'aller chez le médecin (cf. Meillet 1952 : 262-263).

Du point de vue morphologique, on considère généralement que l'ancien français est la première langue à avoir créé un futur synthétique. Le futur italo-roman résulte donc de la fusion ou de l'agglutination de l'infinitif avec l'auxiliaire habere. A partir de venire + habeo, on aurait donc eu *venirabjo > *veniraßjo > *venrajo > *verrajo > verraggio " je viendrai ", formes attestées dans les textes anciens. On peut citer pour divers dialectes les formes suivantes (cf. Rini 1995 : 423) :

(3)

Toscan ancien : diragio, risapraggio, faraggio

Siénois ancien : faragio, moragio, vorragio

Ombrien ancien: daràgio, daràio

Romain ancien: darajo, dicerajo

Aquilano ancien : contaragio

Napolitain ancien : porragio

Sicilien ancien : truviraju, mustriraiu

Napolitain littéraire : cantarraggio

Ombrien moderne : troerajju

Calabrais moderne : canteraju, amaroggiu

Sicilien moderne : faroggiu, viniroggiu

Dans les formes antiques du type diragio «je dirai », faragio «je ferai » (siénois ancien) qu'on rencontre chez Brunetto Latini, on identifie donc parfaitement l'auxiliaire aggio; de même dans les formes dirabbo "je dirai ", farabbo "je ferai », metterabbo "je mettrai », formées à partir de abbo « j’ai » (Rohlfs $1968: 272, \$ 541$ et 332, §587).

Notons que dans les formes contemporaines telles que dormirò «je dormirai », farò «je ferai », etc. l'auxiliaire, réduit à un affixe flexionnel, n'est plus identifiable comme un 
élément autonome. On a donc affaire à un phénomène d'univerbation, i.e. un processus de fusion de deux éléments originellement distincts. Ce type d'évolution en vertu duquel, après une phase d'oscillation, un auxiliaire tombe au statut d'affixe - et plus particulièrement de suffixe - a pu être mis sur le compte de la préférence suffixale des langues romanes (cf. Klausenburger $2000: 155$ ). Le même type de fixation s'est produit en espagnol après une phase d'oscillation. Ainsi d'après Rini (1990: 359), «In Old Spanish, when the morpheme é was free, it was not only detachable from the verb, but it lacked a fixed position in relation to the verb. For example, we find constructions like amar é, é de amar, and é amar. However, once the free morpheme é becomes bound, it takes on a fixed position, e.g., amaré not *eamar $»^{5}$.

Ajoutons que les formes du type vorraggio « je voudrai », moraggio « je mourrai », diraggio « je dirai », etc. et celles du type vorrò, morirò, dirò, etc. doivent être considérées comme distinctes. Dans les premières en effet, c'est la voyelle correspondant à l'ancien auxiliaire qui est tonique (cf. ['addzo]). Le -ò de vorrò, morirò, dirò ne peut donc pas représenter le -o final de vorraggio, moraggio, diraggio. Une hypothèse possible est de voir dans le -ò final du futur un -o analogique des formes fréquentes telles que sto, so, do, etc (cf. Rini 1995 : 422). Les deux personnes du pluriel des verbes «parler » et «faire " quant à elles n'ont pas * parleravemo / faravemo ou parleravete / faravete, mais *parleremo / faremo ou parlerete / farete. De toute évidence les formes en -emo et -ete sont des réductions de avemo / avete.

\section{Le futur en sarde}

Contrairement à l'italien et d'autres variétés romanes, le sarde connaît non pas un futur synthétique mais un futur analytique fondé sur la périphrase haběo ad cantāre. Comme l'illustrent les tableaux (1-2) suivants basés sur les cartes 653 et 1146 de l'Atlante Italo Svizzero (AIS), il s'agit d'une construction pan-sarde ${ }^{6}$.

Tableau 1

\begin{tabular}{|c|c|c|c|c|c|c|}
\hline & \multicolumn{6}{|c|}{ Question 653 : Non dormirò « je ne dormirai pas» } \\
\hline 990 & Sant'Antioco & & nว & 'app & $\mathrm{a}$ & dro'mi \\
\hline 985 & Cagliari & & non & & & 'dormu \\
\hline 973 & Villacidro & & no & app & $\mathrm{a}$ & ddro'mi \\
\hline 968 & Perdasdefogu & & non & 'app & a & ddro'miri \\
\hline 967 & Escalaplano & 'd $\varepsilon u$ & non & & & 'drommu \\
\hline 963 & Mogoro & 'd $\varepsilon \mathrm{u}$ & non & & & 'drommu \\
\hline 959 & Baunei & 'dzeo & nวn & & & 'drommo \\
\hline 957 & Desulo (Nuoro) & & non & & & 'drommo \\
\hline 955 & Laconi (Oristano) & 'dعo & no & 'app & $a$ & ddro'miri \\
\hline
\end{tabular}




\begin{tabular}{|c|c|c|c|c|c|c|}
\hline 954 & Busachi (Oristano) & & non & & & 'dormo \\
\hline 949 & Dorgali (Nuoro) & & no & 'app & a & ddro'mire \\
\hline 947 & Fonni (Nuoro) & & non & 'app & a & ddro'mire \\
\hline 943 & Macomer (Nuoro) & & no & 'app & a & dro'mire \\
\hline 942 & Santu Lussurgiu (Oristano) & & non & & & 'drommo \\
\hline 941 & Milis (Oristano) & & no & 'app & a & ddro'miri \\
\hline 938 & Bitti (Nuoro) & 'jعJ & non & & & 'dormo \\
\hline 937 & Nuoro & & non & & & 'dormo \\
\hline 923 & Ploaghe (Sassari) & & no & 'app & a & ddrom'mire \\
\hline 922 & Sassari & & no & 'voKKu & & drum'mi \\
\hline 916 & $\begin{array}{l}\text { Tempio } \\
\text { (olbia Tempio) }\end{array}$ & & no & & & 'drommu \\
\hline
\end{tabular}

Tableau 2

\begin{tabular}{|c|c|c|c|c|c|c|c|c|}
\hline & Question 1146 : Le & domani & “je le & s vendra & i dem & & & \\
\hline 990 & Sant'Antioco & & & a'duzu & & & 'vendu & 'kras \\
\hline 985 & Cagliari & & & 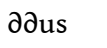 & & & 'bendu & 'kras \\
\hline 973 & Villacidro & 'krazi & & 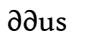 & 'app & $\mathrm{a}$ & 'vendi & \\
\hline 968 & Perdasdefogu & & $\mathrm{d} \varepsilon \mathrm{u}$ & 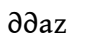 & 'app & $\mathrm{a}$ & $b^{\prime} b \varepsilon n d i$ & 'kras \\
\hline 967 & Escalaplano & & & 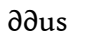 & 'app & a & 'vendi & 'kras \\
\hline 963 & Mogoro & & & $\partial^{\prime} \partial u z^{u}$ & & & 'vendu & 'kraz ${ }^{\mathrm{i}}$ \\
\hline 959 & Baunei & & & 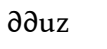 & 'app & $\mathrm{a}$ & 'vendere & $\mathrm{kraz}^{\mathrm{a}}$ \\
\hline 957 & Desulo (Nuoro) & & & даว & & & b'bennว & 'kraz ${ }^{\mathrm{a}}$ \\
\hline 955 & Laconi (Oristano) & & & 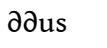 & 'app & $\mathrm{a}$ & ' $\beta \varepsilon n d \varepsilon$ & 'kras \\
\hline 954 & Busachi (Oristano) & & & $\partial^{\prime} \partial u z^{u}$ & & & 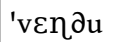 & 'kraz ${ }^{\mathrm{a}}$ \\
\hline 949 & Dorgali (Nuoro) & & & $\operatorname{loz}$ & 'app & $\mathrm{a}$ & $\mathrm{v}^{\prime} \mathrm{v} \varepsilon \eta \partial \varepsilon$ & $\mathrm{k}^{\prime} \mathrm{kras}$ \\
\hline 947 & Fonni (Nuoro) & & & $\operatorname{loz}$ & 'app & $\mathrm{a}$ & 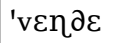 & $\mathrm{k}^{\prime} \mathrm{kras}$ \\
\hline
\end{tabular}




\begin{tabular}{|c|c|c|c|c|c|c|}
\hline 943 & Macomer (Nuoro) & lor & & & 'ßعпдо & 'kras \\
\hline 942 & Santu Lussurgiu (Oristano) & 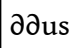 & 'app & a & ' $\beta \varepsilon n n \varepsilon r$ & 'kras \\
\hline 941 & Milis (Oristano) & 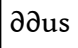 & 'app & a & bbendi & $\mathrm{kraz}^{\mathrm{a}}$ \\
\hline 938 & Bitti (Nuoro) & lor & & & 'vعпةว & 'kraz ${ }^{\mathrm{a}}$ \\
\hline 937 & Nuoro & lor & & & 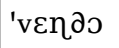 & 'kraza \\
\hline 923 & Ploaghe (Sassari) & loz & 'app & a & 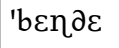 & 'xras \\
\hline 922 & Sassari & li & & & 'vendu & du'mani \\
\hline 916 & Tempio (Olbia Tempio) & li & 'adzdzu & a & vin'di & du'mani \\
\hline
\end{tabular}

17 Les tableaux en (1-2) montrent également que dans un certain nombre de dialectes, la forme utilisée pour exprimer le futur est clairement une forme de présent, ce qui de toute évidence est à mettre au compte de la valeur neutre ou non-marquée de ce "temps »d'où son aptitude à occuper n'importe quelle position dans le spectre temporel - et ce qui illustre l'enracinement du futur dans le hic et nunc du moment de locution. Précisons enfin qu'en sassarese et en gallurese au nord de l'île, le futur est clairement synthétique (cf. sass. [kanta'raddzu], gall., [kanta'raggju] (Blasco Ferrer 2002: 109)), ce qui constitue un argument supplémentaire pour séparer ces variétés du diasystème sarde proprement dit.

\subsection{Caractéristiques historiques et fonctionnelles du futur en sarde}

D'après Pisano (2009: 148), il semblerait que le sarde n'ait pas connu autre chose qu'un futur analytique, même si Nocentini (2001: 390) signale des tours tels que timerhàpo «je craindrai » et chez l'Araolla cantarhapo « je chanterai », fagherhàt « il / elle fera », etc. A vrai dire, ces formes seraient plus ou moins des créations artificielles de nature littéraire (et limitées à la première personne du singulier) et il est loin d'être clair qu'elles aient jamais été réellement en usage (Pisano, c.p.). D’après Blasco Ferrer (2002: 429), la « conservation » du futur analytique en sarde serait un indice d'archaïcité qui unirait le sarde et d'autres variétés des aires latérales ou isolées de la Romania (le roumain, le portugais ou le rhétoroman), alors que le futur synthétique du sassarese et du gallurese constituerait une innovation.

Le même type de futur est attesté dans les Pouilles, en Sicile (cf. aviri a + infinitif (cf. Bentley (1998)), dans les Abruzzes et en Lucanie, où la construction conserve d'après Loporcaro (1999) une valeur déontique. Ajoutons que le type haběo cantāre est attesté en sarde médiéval (Blasco Ferrer 2003 : 214); il est remplacé aux XVe et XVI ${ }^{e}$ siècles par le tour haběo ad cantāre (cf. Nocentini 2001 : 375) - l'un comme l'autre présentent dès les premières attestations des valences aussi bien temporelles que modales. Dans le Condaghe di San Pietro in Silki et dans les Statuti Sassaresi par exemple, on relève des exemples tels que les suivants, où la valeur déontique de la construction périphrastique affleure assez clairement (cf. Blasco Ferrer 1984 : 110) :

(4) 
a) progitteu ti appo battuier destimonios? (CSP, 194) (litt.: «pourquoi dois-je t'apporter des témoins?»).

b) et si alcunu aet comporare petha e aet esser pesata... (StSS, I, 30/3r) (« ... et si quelqu'un doit / devait acheter de la viande et si celle-ci doit / devait être pesée... »)

En sarde moderne en revanche, on considère que le futur périphrastique ne véhicule plus la valeur déontique qu'il offre dans les textes médiévaux (cf. Jones 1993 : 90), même si à vrai dire ici ou là il semblerait que ce type de lecture soit malgré tout possible, ainsi que le suggère Pisano (2007 : 106) entre autres pour le dialecte de Pula :

(5)

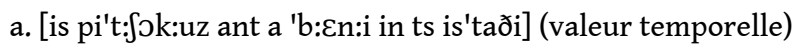

« Les garçons viendront (litt. 'ont à venir') en été »

b. [az a 'n:ai s:u үi 'nau 'd:cu] (modalité jussive)

"Tu diras (litt. 'tu as à dire') ce que je dis moi »

c. [ant 'Es:i is 'tfinku d:e 'үit:si] (modalité épistémique)

«C'est sans doute (litt. 'ont (à) être') cinq heures du matin »

Bentley (1999), qui a consacré toute une étude à la construction áere $a+$ infinitif en sarde, suggère que ce type aurait pris le dessus sur le type áere + infinitif du fait du prestige de l'italien littéraire et du toscan, idée que l'on retrouve chez Meyer-Lübke (1895 : 152-153).

Précisons d'une part que les premières attestations du tour áere $a+$ infinitif remontent au XIII ${ }^{e}$ siècle, à un moment où la domination espagnole en Sardaigne n'était pas établie. On ne peut donc pas l'attribuer au superstrat espagnol. D'autre part, quand l'infinitif commence par une voyelle et notamment par un [a], la préposition peut être absorbée par l'initiale vocalique de l'infinitif (cf. appo (a) andai... « j'irai.... »)

\subsection{Propriétés et contraintes distributionnelles du futur périphrastique}

Le verbe áere fonctionne comme un auxiliaire au regard des clitiques et la cohésion de la séquence áere $a+$ infinitif se manifeste dans un certain nombre de contraintes (cf. Pisano 2009: 151-152) :

(6)

a) les clitiques précèdent en effet le verbe áere et ne sauraient s'interposer entre la préposition et l'infinitif (cf. 'l appo a f'fayere « je le ferai » vs. *'appo a lu 'vayere) (Jones $1988: 175$ et $1993: 146)$. Ce critère a amené un certain nombre de chercheurs à considérer que l'on avait affaire là à une «monoclausal construction » (cf. Jones $1988: 175-176)$.

b) la négation de phrase («sentential negation ») doit précéder l'auxiliaire áere et ne saurait apparaitre devant l'infinitif (cf. no l'l appo a f'fayere «je ne le ferai pas » vs. * 'l appJ a no f'fayere) (Jones, op. cit.) ; étant donné que la négation porte sur la proposition, l'impossibilité de * 'l appo a no f'fayere montrerait que le verbe à l'infinitif ne forme pas une proposition indépendante.

c) les adverbes ne peuvent pas non plus s'interposer entre l'auxiliaire 'appo et la préposition; l'unique option est de placer l'adverbe après l'infinitif: 'l appo a f'fayes 'kraza « je le ferai demain » (cf. * 'l appo 'ðraza a f'fayere).

Il n'y a guère que des marqueurs tels que 'magku «même pas » qui - marginalement puissent rompre la relation entre l'auxiliaire et l'auxilié : no l'l appo 'mayk a salu'Jare «je ne le saluerai même pas» (cf. (7a)). Il convient cependant de préciser qu'il est au moins marginalement possible de factoriser l'auxiliaire dans une structure coordonnée. En l'occurrence une phrase telle que 'amuz a bbuf'fare $\varepsilon$ a mani'yare 'kalki 'kJza «nous boirons et mangerons quelque chose» en ( $7 \mathrm{~b})$ est envisageable, preuve que la 
construction analytique conserve encore l'individualité de ses parties et que de ce point de vue elle ne présente pas le même degré de cohérence et de coalescence que l'on rencontre typiquement dans la construction analytique des formes composées.

(7)

a) no l'l appo 'mayk a salu'ðare

«Je ne le saluerai même pas »

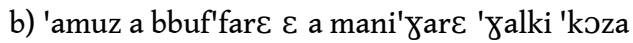

"Nous boirons et mangerons quelque chose"

c) 'amuz a ttor'rare a b'bennere

"Nous reviendrons"

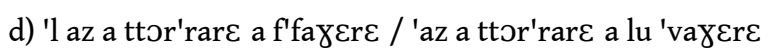

« Tu le referas»

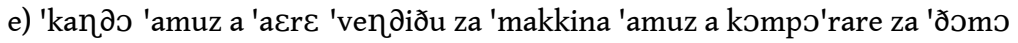

"Quand nous aurons vendu la voiture, nous achèterons la maison »

Ajoutons également que pour exprimer l'itération, plutôt qu'un préfixe on utilise en sarde le tour analytique tor'rare a "revenir à " + verbe à l'infinitif. Or, comme l'illustre l'exemple (7c), l'auxiliaire prend pour complément non pas le verbe lexical 'bEnnere «venir» porteur de l'information sémantique principale, mais le verbe tor'rare a. Néanmoins c'est bien le verbe principal qui assigne les rôles sémantiques, comme en témoigne la construction transitive en (7d), où le clitique objet précédant l'auxiliaire áere

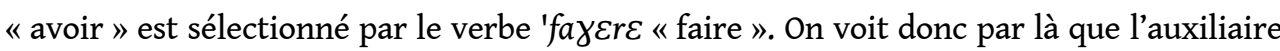
áere "avoir " avec lequel se construit le futur jouit d'une certaine mobilité et d'une certaine indépendance au regard de l'auxilié. Et la même mobilité caractérise le clitique objet masculin singulier lu qui peut apparaître soit à gauche de l'auxiliaire $a z$ «tu as », soit à gauche de l'auxilié 'fayere (cf. (7d)).

\subsection{Le futur et les interrogatives}

Les constructions interrogatives fournissent des indications intéressantes concernant le degré de coalescence ou de figement de la construction áere a + infinitif. Rappelons que le sarde recourt à diverses stratégies pour exprimer ce que Tesnière désigne comme interrogation connexionnelle et interrogation nucléaire. En-dehors de l'intonation qui suffit à elle seule à marquer un énoncé comme interrogatif, le sarde utilise d'une manière privilégiée le fronting et le marqueur a (< lat. aut), c'est-à-dire ou bien (cf. Wagner 1984 : $34-35 \$ 17$ et $332 \S 370$; Pittau 1972: 37-38, 143-145) -- et apparait en position initiale comme marqueur de l'interrogation connexionnelle. Les exemples (8)-(10) fournissent une illustration de la syntaxe de ce marqueur :

(8)

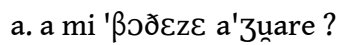

«Est-ce que tu peux m'aider?»

(9)

a. an'nع, a nos 'kumbiðaza?

«Annedda, tu nous invites?»

b. *a an'nع, nos 'kumbiðaza?

(10)

a. u'n atteru Yaf'f $\varepsilon$, a mi lu 'atizi ?

«Un autre café, tu me l'apportes?»

b. *a u'n atteru Xaf'f $\varepsilon$, mi lu 'atizi ?

L'exemple (8a) montre que la particule $a$ précède en principe la proposition qui fait l'objet du questionnement ${ }^{7}$. L'exemple (9a) quant à lui illustre la portée de ce marqueur : le 
vocatif Anne' étant à l'extérieur de la prédication nucléaire, il ne peut se trouver dans le champ du marqueur $a$; la même observation vaut évidemment pour l'exemple (10a) : la particule $a$ ne peut pas précéder le SN thématique un atteru café; elle doit nécessairement occuper la position initiale de la proposition nucléaire sur laquelle elle porte.

L'autre stratégie à laquelle recourt le sarde est celle du fronting - il convient du reste de préciser qu'il s'agit d'un trait de la syntaxe sarde qui dépasse largement le contexte des interrogatives (la même stratégie est utilisée dans les exclamatives : cf. bellu est!! « Qu'il est beau! »). Les exemples en (11) présentent un échantillon des catégories qui dans les interrogatives sont susceptibles d'être antéposées :

(11)

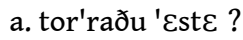

«Il est revenu?»

b. fa'miðu 'zez\& ?

«Tu as faim?»

c. kum'prezu 'm aza?

«Tu m'as compris?»

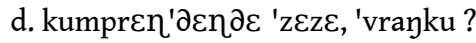

«Tu comprends, Franck?»

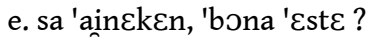

« La Heineken, elle est bonne?»

L'élément susceptible d'être placé en position initiale de proposition peut être de nature variable ; il peut s'agir d'un participe passé (11a-c) ; il peut s'agir d'un gérondif (11d), ou même d'un adjectif (11e). En revanche, et c'est le point essentiel, le fronting est précisément exclu avec le futur périphrastique (cf. (12a)) ainsi qu'avec le conditionnel (cf. (12c)) qui est lui aussi périphrastique (voir Jones $1988: 185$ et $1993: 147)$ :

(12)

a. * a lu 'vayere, 'aza?

«Est-ce que tu le feras?»

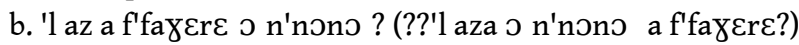

«Tu le feras ou non?»

c. * a lu 'vayere, 'tiaza

«Est-ce que tu le ferais? ? (cf. a lu ðias fayere?)

Autrement dit dans l'exemple en (12a), la cohésion entre l'auxiliaire et l'auxilié est rompue, d'où l'agrammaticalité des séquences qui les dissocient en antéposant l'infinitive mais en laissant l'auxiliaire en position post-verbale.

Il faut ajouter que dans les structures interrogatives disjonctives en "ou non? ", la négation autonome (holophrastique) 'nગnว «non» peut difficilement s'insérer après

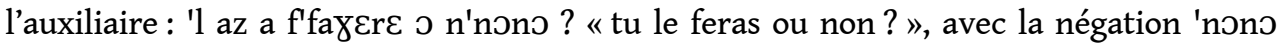
après l'infinitif, est l'unique option pour signifier la mise en question du contenu exprimé par le tour périphrastique. Un énoncé tel que ?? 'l aza 0 n'nono a f'fayere? (« tu le feras ou non?») est donc exclu. De ce point de vue il y a une grande différence entre les constructions qu'on vient de présenter et les structures modal + infinitif qu'on trouve aussi en français: Le médecin de garde vous indiquera si vous devez ou non vous rendre à l'hôpital. Dans ce type d'exemple en effet, il est possible d'insérer le tour disjonctif soit à droite de l'auxiliaire modal, soit à droite de l'auxilié, i.e. la forme infinitivale qui porte l'information lexicale du tour (cf. Le médecin de garde vous indiquera si vous devez vous rendre ou non à l'hôpital). Naturellement, il est possible aussi d'insérer le tour disjonctif à la fin de la proposition subordonnée (cf. Le médecin de garde vous indiquera si vous devez vous rendre à l'hôpital ou non). 
ànt à l'exemple (12c), il montre que le sarde recourt au verbe dévere "devoir " à l'imparfait suivi de l'infinitif pour exprimer le conditionnel. Aussi convient-il de préciser que la formation du futur périphrastique est soumis à toute une série de contraintes morphonologiques dont il convient de dire quelques mots.

\subsection{La morphonologie du futur en sardo logudorese}

Le sardo logudorese forme le futur analytique selon un patron qui correspond à celui du sarde en général. Le dialecte de Buddusò par exemple recourt à l'auxiliaire áere suivi de la préposition $a$ et de l'infinitif (cf. Molinu 1988-89: 70) :

(13)

a. 'l az a f'fayere

« Tu le feras"

b. 'appo a kkan'tare

«Je chanterai »

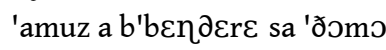

« Nous vendrons la maison»

Ajoutons que les auxiliaires éssere «être » et áere «avoir » font leur futur en recourant non pas à l'auxiliaire áere mais à l'auxiliaire dévere « devoir » (cf. également Pittau 1972 : 92 pour des formations analogues en Nuorese). Comme l'illustre l'exemple (14a), l'auxiliaire dévere " devoir » est cantonné à la formation du futur de ces verbes-là. Les exemples (14b-d) montrent que le logudorese (variété de Buddusò) recourt à l'imparfait de dévere pour former le conditionnel (cf. Pittau 1972: 91-92; Molinu 1988-89: 84 et 1999: 134 ; Blasco Ferrer $2002: 86$, etc.) :

(14)

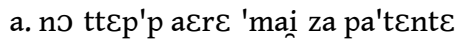

"Je n'aurai jamais le permis de conduire »

b. 'tia mani'yare

« Je mangerais"

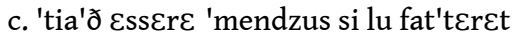

"Ce serait mieux s'il / elle le faisait »

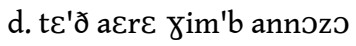

« Il / elle doit avoir 5 ans »

e. $t \varepsilon^{\prime} \partial$ a $\varepsilon^{\prime} r$ appidu Xim'b annozo

«Il / elle devait avoir 5 ans »

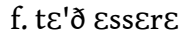

«Probablement... »

Pour ce qui est des exemples (14d-f), ils montrent que la construction analytique se teinte d'une valeur modale probabilitative évidente qui est tout à fait conforme au contenu virtuel ou potentiel du futur. C'est du reste là une asymétrie fondamentale entre ces deux temps que sont le préterit (passé composé) et le futur : si l'un et l'autre s'enracinent dans le présent de l'acte de parole, la mise en acte de l'état de fait décrit par l'énoncé au futur demeure dans le domaine de l'inactuel et du prospectif, ainsi que l'avait brillamment énoncé Kuryłowicz (1975b : 182).

Le point à souligner est que, y compris pour les verbes éssere et áere, la construction avec áere a + infinitif est également disponible (cf. appo a éssere "je serai ", az a éssere " tu seras », etc.). D'autre part et surtout, la forme du verbe dévere "devoir» utilisée pour former le futur et le conditionnel des auxiliaires est formellement distincte de celle du verbe plein correspondant (cf. Molinu 1988-89 : 71). 
(15)

a. présent imparfait b. présent imparfait

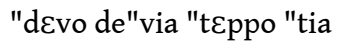

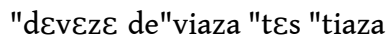

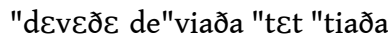

de"vimuzu de"viamuzu "temus ti"amuzu

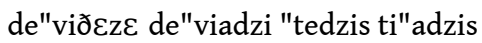

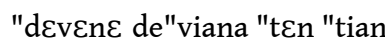
substance phonétique, selon un principe bien connu par ailleurs et que Rini (1999: 122) rappelle en ces termes: "When any element becomes a dependent grammatical morpheme, there is a general tendency for that element to undergo phonetic attrition ». En outre, en tant qu'auxiliaire le verbe dévere « devoir » présente un assourdissement de la consonne initiale qui n'est pas non plus régulier. Ce dernier constitue un phénomène de fortition consonantique qui n'affecte que l'auxiliaire (cf. Molinu 1988-89: 60). Encore une fois le caractère déviant de cette forme est à mettre au compte de son statut d'auxiliaire.

\subsection{Le cas du jussif périphrastique}

On signalera enfin une construction analytique du plus grand intérêt qui elle aussi repose sur le tour a + infinitif. Il s'agit en l'occurrence d'une construction jussive qui n'est pas sans rapport avec la construction utilisée pour exprimer le futur. Comme l'illustrent les exemples (16), la valeur directionnelle d'éloignement par rapport à un point de référence n'est sans doute pas étrangère aux nuances que la construction est susceptible d'assumer (cf. Molinu 1988-89 : 68, 93) :

(16)

a. a lu 'vaðعre (

«Fais-le / qu'on le fasse !»

b. a bbos is'tare 'XjettJzo

« Restez tranquilles!»

c. - a kken't annozo !

«A cent ans !» (= « puisses-tu vivre jusqu'à cent ans »)

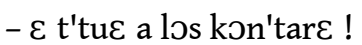

«Et toi à les compter !» (= « et toi puisses-tu les compter !»)

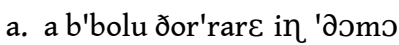

«Qu'on puisse vous le rendre !»

a. a nnol 'viðعs 'sanozว

«Au revoir!» (= «à nous voir sains ») (cf. Wagner $1997: 338)$

Ces constructions (qui ne sont pas sans rappeler le type «A ver!» de l'espagnol), confirment le point de vue de Kuryłowicz (1975b : 182) d'après lequel le paramètre du moi-ici-maintenant constitue une donnée fondamentale du langage et la source fondamentale de renouvellement des catégories grammaticales. C'est à ce principe qu'il convient d'imputer la promotion de formes de futur pour l'expression de l'impératif dans certains dialectes des Andes équatoriales (cf. Harris 1998 : 33) : 
(17)

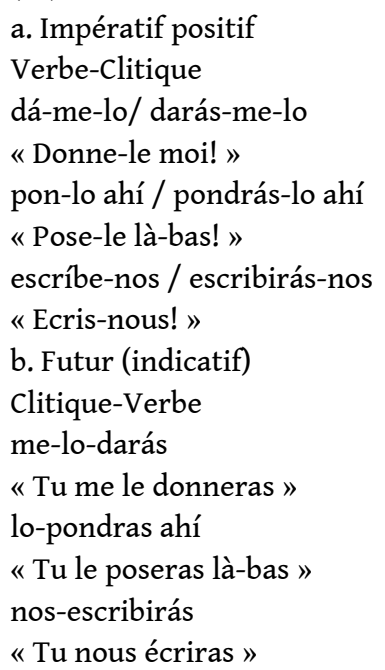

51 A vrai dire d'après les données de Harris l'injonction directe peut être exprimée soit par des formes d'impératif dédiées, soit par des formes qui morphologiquement sont des formes de futur et dont on peut observer qu'elles impliquent l'enclise des clitiques. Dans tous les cas les données des dialectes des Andes illustrent si nécessaire le principe de Rasmus Rask (1824/2001 : 158-159) d'après lequel l'impératif et le futur sont étroitement liés.

\subsection{La question de l'analycité}

On a pu considérer que le passage du latin aux langues romanes illustre le passage d'une syntaxe fondamentalement synthétique - celle du latin - à une syntaxe fondamentalement analytique - celle des langues romanes. Telle est par exemple la position de Tekavcic (1980: 15). Or, comme le souligne Schwegler (1990: 28), les adjectifs «synthétique » et "analytique » caractérisent des constructions bien plus que des langues. Tekavcic (1980: 15) considérait du reste le latin comme "prevalentemente sintetico» («dont le synthétisme est prédominant». Et ces constructions peuvent afficher des degrés variables d'analyticité ou de synthèse. Vincent (1996:100) observe que même si le latin a pu être considéré comme étant fondamentalement synthétique, la négation non y a une forme analytique, les périphrases verbales n'y sont pas inexistantes (cf. esse + participe passé) et les prépositions y sont très largement utilisées. Et il ne faut pas oublier non plus qu'un certain nombre de formations dites "synthétiques » du latin sont diachroniquement des constructions analytiques, comme c'est le cas en l'occurrence du futur amābo qu'on fait remonter à un tour périphrastique *[ama] + *bhū « je suis / deviens » où la base verbale a fusionné avec l'auxiliaire " être ». On a affaire de ce point de vue à un développement en spirale en vertu duquel une construction qui était analytique se synthétise, puis l'érosion phonétique ou le besoin d'expressivité amènent une nouvelle construction analytique qui à son tour va finir par s'agglutiner via un phénomène d'univerbation, et ainsi de suite (cf. Vincent 1996 : 105).

Si donc on s'en tient aux constructions, on pourra en effet remarquer que le sarde peut dire pjul 'vonu et pjus 'payu (mot à mot «plus peu ») là où le français et l'italien ont respectivement meilleur ou migliore et moins / meno. On a vu plus haut qu'au lieu de recourir à un préfixe par exemple pour exprimer l'itérativité, le sarde recourt à une construction complexe constituée du tour torrare a "retourner à » + infinitif qui, 
employée au futur, met en jeu une structure prépositionnelle double. On pourra remarquer aussi que le sarde connaît un accusatif prépositionnel, lequel recourt donc à des prépositions pour marquer l'objet direct humain / animé / défini (cf. Floricic 2003). Et les prépositions sont également sélectionnées pour exprimer le dépendant d'une tête qui peut être explicite ou pas : s'amigu e Pedru «l'ami de Pedru» / su (x) e Pedru. Ajoutons enfin une caractéristique originale du point de vue de l'expression de la temporalité et du concept de "marque ». Là où une langue comme le français utiliserait le présent de l'indicatif, le sarde recourt plus volontiers et plus systématiquement à une construction

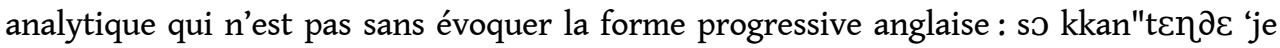
chante' (cf. Blasco Ferrer 1984 : 252). L'extension fonctionnelle de ce présent progressif est également attestée en anglais, où précisément elle n'est plus utilisée pour indiquer simplement la coïncidence entre la référence de l'événement et celle du moment de locution, mais la référence à un événement postérieur au moment de locution (cf. I'm coming tomorrow).

\section{Conclusion}

Bybee (1985 : 158) observait que le futur était relativement instable en tant que temps grammatical. L'évolution diachronique porte en effet au renouvellement continuel des formes de futur, d'où l'émergence de formes et de constructions qui peuvent être alternativement synthétiques ou analytiques (cf. Bybee 1985 : 158). La distinction même entre analytisme et synthétisme doit être considérée avec prudence. L'originalité du sarde ne réside pas seulement dans le fait qu'il connait un tour analytique / périphrastique pour l'expression régulière du futur. Elle réside dans le fait que ce tour s'inscrit dans une constellation de constructions qui sont elles aussi éminemment analytiques. L'intérêt du futur périphrastique est qu'il se situe d'une certaine manière à mi-chemin entre la morphologie et la syntaxe. De ce point de vue, et compte tenu des contraintes distributionnelles qu'on a évoquées, la question de savoir s'il convient de considérer les formes périphrastiques de futur sur le même plan que les formes flexionnelles intégrées dans des paradigmes morphologiques est loin d'être évidente. Peut-être serait-il plus judicieux, à l'instar de Šabršula (1966), de considérer les constructions périphrastiques dont il a été question ici comme des formations prémorphologiques.

\section{BIBLIOGRAPHIE}

ANDERSEN, H., 2010, « From Morphologization to Demorphologization », dans S. Luraghi \& V. Bubenik, The Continuum Companion to Historical Linguistics, Continuum, London, New York, p. 117-146

BENTLEY, D., 1998, « Modalità e tempo in siciliano: un'analisi diacronica dell'espressione del futuro ", Vox Romanica, 57, p. 117-137 
BENTLEY, D., 1999, « On the origin of Sardinian aere a plus infinitive », Medioevo Romanzo, 23 (3), p. $321-358$

BENVÉNISTE, E., 1966, Problèmes de linguistique générale 1, Gallimard, Paris

BLASCO FERRER, E., 1984, Storia linguistica della Sardegna, Niemeyer, Tubingen

BLASCO FERRER, E., 1986, La lingua sarda contemporanea, Ed. Della Torre, Cagliari

BLASCO FERRER, E., 2002, Linguistica sarda. Storia, metodi, problemi, Condaghes, Cagliari

BLASCO FERRER, E., 2003, Crestomazia sarda dei primi secoli. Tesi, grammatica storica, glossario, Ilisso, Nuoro (Officina Linguistica 4)

BONFANTE, G., 1998, The Origin of the Romance Languages: Stages in the Development of Latin, Universitätsverlag C. Winter, Heidelberg (Bibliothek der klassischen Altertumswissenschaften 100) BRINTON, L. J., CLOSS TRAUGOTT, E., 2005, Lexicalization and Language Change. Cambridge University Press, Cambridge, New York

BYBEE, J. L., 1985, Morphology: A study of the relation between meaning and form, John Benjamins, Amsterdam / Philadelphia

FLORICIC, F., 2003, « Notes sur l'accusatif prépositionnel en sarde », Bulletin de la Société de Linguistique de Paris, 98 (1), p. 247-303

HAIMAN, J., 1971, « Targets and Paradigmatic Borrowing in Romantsch », Language 47 (4), p. 797-809

HARRIS, J., 1998, « Spanish imperatives : syntax meets morphology », Journal of Linguistics, 34, p. 27-52

HERMAN, J., 2006, « Remarques sur l'histoire du futur latin - et sur la préhistoire du future roman ", dans Du latin aux langues romanes II. Nouvelles études de linguistique historique réunies par Sándor Kiss avec une préface d'Alberto Varvaro, Max Niemeyer, Tübingen

HERZOG, E., 1906, Neufranzösische dialekettexte mit grammatischer einleitung und wörterverzeichnis, Reisland O. R., Leipzig

JONES M. A., 1988, « Auxiliary verbs in Sardinian », Transactions of the Philological Society 86, p. 173-203

JONES M. A., 1993, Sardinian Syntax. Routledge, London \& New York

KLAUSENBURGER, J., 2000, Grammaticalization. Studies in Latin and Romance morphosyntax, John Benjamins, Amsterdam, Philadelphia

KURYŁOWICZ, J., 1975a, « Linguistics of To-day », dans Esquisses Linguistiques II, Wilhelm Fink Verlag, München, p. 9-29 (Coll. Internationale Bibliothek für Allgemeine Linguistik. Band 37)

KURYŁOWICZ, J., 1975b, « The Role of Deictic Elements in Linguistic Evolution », dans Esquisses Linguistiques II, Wilhelm Fink Verlag, München, p. 121-130 (Coll. Internationale Bibliothek für Allgemeine Linguistik. Band 37)

LAUSBERG, H., 1971, Linguistica Romanza. II. Morfologia, Feltrinelli Editore, Milano

LOPORCARO, M., 1999, « Il futuro cantare-habeo nell'Italia meridionale », Archivio Glottologico Italiano, 84 (1), p. 67-114

MEIER, H., 1965, « Futuro y futuridad », Revista de Filología Española, 48 (1/2), p. 61-77 
MEILLET, A., 1948, « L'évolution des formes grammaticales », dans Linguistique historique et linguistique générale, Honoré Champion, Paris, p. 130-148

MEILLET, A., 1952, Esquisse d'une histoire de la langue latine, Klincksieck, Paris

MEYER-LÜBKE, W., 1895, Grammaire des langues romanes. T.2. Morphologie, H. Welter, Paris

MikLosich, F., 1861, « Die slavischen Elemente im Rumunischen », Denkschriften der Kaiserlichen

Akademie der Wissenschaften, Philosophisch-Historische Klasse, 12 (1), p. 1-70

MOLINU, L., 1988-1989, Morfologia verbale del buddusoino (Varietà logudorese di Buddusò), Università di

Pisa

MOLINU, L., 1999, « Morfologia logudorese », dans Bolognesi, R., \& Helsloot, K., (éds.), La lingua

sarda. L'identità socioculturale della Sardegna nel prossimo millennio. Atti del Convegno di Quartu

Sant'Elena, 9-10 maggio 1997, Condaghes, Cagliari, p. 127-136

NOCENTINI, A., 2001, «La genesi del futuro e del condizionale sintetico romanzo », Zeitschrift für

Romanische Philologie, 117, p. 366-403.

PISANO, S., 2007, Il sistema verbale del sardo moderno: tra conservazione e innovazione, Edizioni ETS,

Pisa

PISANO, S., 2009, « Il futuro e il condizionale analitici in alcune varietà sarde moderne: Genesi di marche grammaticali da forme verbali lessicalmente piene », Bollettino di Studi Sardi, 2, p. 147-166

RASK, R., 2001 [1824], Gramática Española según un nuevo plan (1824). Edición y estudio preliminar de Josefa Dorta, Arco Libros, Madrid

RINI, J., 1990, « Dating the grammaticalization of the Spanish clitic pronoun », Zeitschrift für romanische Philologie, 106, p. 354-370.

RINI, J., 1995, « Syntactic and pragmatic factors in the morphological reduction of Latin HABEO > Spanish (h)e », Neophilologus, 79, p. 421-432

RINI, J., 1999, Exploring the Role of Morphology in the Evolution of Spanish, John Benjamins, Amsterdam, Philadelphia

ROHLFS, G., 1968, Grammatica Storica della Lingua Italiana e dei suoi Dialetti. Morfologia, Einaudi, Torino (Coll. Piccola Biblioteca Einaudi 149)

ŠABRŠULA, J., 1966, « Un problème de la périphérie du système morphologique: à propos des formations prémorphologiques ", Travaux Linguistiques de Prague 2. Les problèmes du centre et de la périphérie du système de la langue, Academia, Prague. p. 183-192.

SCHWEGLER, A., 1990, Analyticity and syntheticity. A diachronic perspective with special reference to Romance languages, de Gruyter, Berlin.

TEKAVČIĆ, P., 1980, Grammatica storica dell'italiano. II. Morfosintassi, III. Lessico, Il Mulino, Bologna TESNIÈRE, L., 1939, « Théorie structurale des temps composés », dans Mélanges de linguistiques offerts à Charles Bally, Georg et Cie, Genève, p. 153-183

THACKSTON, W. M., 2006, Sorani Kurdish - A Reference Grammar with Selected Readings.

томіс́, O. M., 2006, Balkan Sprachbund. Morphosyntactic Features, Springer, Dordrecht

VÄÄNÄNEN, V., 2006, Introduction au latin vulgaire, Klincksieck, Paris

VINCENT, N., 1996, « Synthetic and Analytic Structures », dans Maiden M., \& Parry, M. (éds), (1996), The Dialects of Italy, Routledge, London, p. 99-105. 
WAGNER, M. L., 1938, «Flessione nominale e verbale nel sardo antico e moderno », L'Italia Dialettale ,14, p. 93-170

WAGNER, M. L., 1941, Historische Lautlehre des Sardischen, Max Niemeyer, Halle (trad. it. de Giulio Paulis avec introduction et appendice, Fonetica storica del sardo. Gianni Trois Editore, Cagliari 1984)

WAGNER, M. L., 1997, La lingua sarda. Storia, spirito e forma, Ilisso, Nuoro

\section{NOTES}

1. «Le futur de l'indicatif s'exprime par l'intermédiaire du présent de l'infinitif et du présent de l'indicatif du verbe avoir 'j'ai', 'tu as', et ainsi disons-nous j'aimerai comme si nous disions j'ai à aimer ».

2. On peut noter qu'en latin même le verbe habēre pouvait avoir une valeur grammaticale existentielle, ainsi que le rappelle Väänänen $(2006: 128)$ : « Un emploi impersonnel de habet 'il y a' et de continet 'il est écrit', accompagnés d'un complément d'objet, se développe à basse époque : Anthim. 33 avis (illa) bona est, sed puto hic non habere '... mais je crois qu'il n'y en pas ici' ». D'où le point de vue d'Andersen (2010: 146, note 6) d'après lequel le terme de grammaticalisation serait ici inapproprié: "The change of habeo to future auxiliary is traditionally cited as an example of grammaticalization. But note that (i) Lat. habeo 'have' is a verb of existence, i.e., it is a grammatical verb; (ii) the modal habeo + inf. 'have to' is a grammatical verb; it results from a reanalysis (a regrammation) of an extension of habeo 'have'; (iii) habeo + inf. 'future' results from yet another reanalysis (regrammation). The regrammation of 'have to' as 'future' entailed a renewal of the deontic habeo + inf. 'have to' as habeo de/ab + inf., cf. It. avete di ritornare, Fr. vous avez à retourner 'you have to return' " (Cf. aussi Brinton \& Traugott 2005: 24 qui insistent sur le processus de réanalyse et d'unification d'une structure biclausale).

3. La particule $\theta a$ du grec moderne résulte de la fusion / coalescence du verbe "vouloir » thélō et de la conjonction hina « que » (cf. Meillet $1948: 145$ ).

4. Le futur synthétique serait au contraire une innovation assez récente : "Although the future form is common to the Romance languages, it is new in Romantsch itself, having been borrowed from Italian in the last four hundred years. It is never attested in the early texts, dating from the latter half of the sixteenth century, and is still absent in Surselvan, which continues to use a periphrastic future consisting of the auxiliary vegnir 'to come' and the infinitive of the main verb » (Haiman 1971 : 808).

5. Déjà Meyer-Lübke (1895: 152, §112) avait mis en rapport l'alternance cantare habĕo / haběo cantare avec la problématique de l'ordre des mots (cf. aussi Nocentini 2001 : 371-372).

6. Les deux colonnes de gauche indiquent respectivement le numéro du point d'enquête ainsi que le nom de la localité correspondante.

7. Il convient d'insister sur le fait que la particule interrogative $a$ ne doit pas être confondue avec la préposition homophone $a$, l'une et l'autre pouvant d'ailleurs apparaître dans le même énoncé cf. à ce propos des exemples tels que A mamma tua, a l'as bida custu mandzanu? "Ta mère, tu l'as vue ce matin? ", où le premier $a$ introduit l'objet direct en fonction de topic, alors que le second constitue l'exposant de l'interrogation connexionnelle. 


\section{RÉSUMÉS}

L'objectif de cette contribution est d'offrir une description du futur en sardo logudorese. Après avoir rappelé quelques-unes des caractéristiques fonctionnelles et sémantiques essentielles du futur et de sa genèse, l'article présente, sur la base de données dialectologiques existantes (les atlas linguistiques) et d'enquêtes de terrain réalisées en Sardaigne, quelques-unes des spécificités morpho-phonologiques de la formation du futur dans la variété logudorese parlée à Buddusò (Provincia Olbia-Tempio). On verra que pour exprimer le futur cette variété recourt à une construction de nature périphrastique du type "J'ai à + verbe à l'infinitif ». On verra également que d'autres constructions périphrastiques sont attestées en sarde, qui posent naturellement la question de l'analycité telle qu'elle se manifeste dans cette variété.

The aim of this paper is to offer a description of the Future in sardo logudorese. After a presentation of some fundamental properties of the future from the functional and semantic point of view, the paper investigates the morpho-phonological properties of the Future in the Logudorese variety spoken in Buddusò (Provincia Olbia-Tempio). It will be shown that the periphrastic future found in this Sardinian variety resorts to the inflected form of áere 'to have' plus the infinitive of the lexical verb. On the other hand, the Future of the auxiliaries éssere 'to be' and áere 'to have' is formed using the present indicative of the verb dévere 'to have to' followed by the lexical verb in the infinitive. It will be shown as well that other periphrastic constructions are attested in Sardinian, thus raising the question of analyticity as manifested in this variety.

\section{INDEX}

Mots-clés : temps futur, langue sarde, variété sardo logudorese

Keywords : future tense, Sardinian language, Sardo Logudorese variety

\section{AUTEUR}

\section{FRANCK FLORICIC}

Université de Paris III - Sorbonne Nouvelle 